VOL. $3(1970), 265-268$.

\title{
An existence theorem for differential equations
}

\section{J. E. Drummond}

An existence theorem is given for differential equations which fail the simple Lipschitz condition on some surfaces but whose solution curve is not tangential to any of these surfaces.

The differential equation $y^{\prime}=y^{\frac{1}{2}}$ fails the simple Lipschitz condition on the line $y=0$ and solutions through a point on this line are not unique. On the other hand the $\mathrm{DE} y^{\prime}=(y+x)^{\frac{1}{2}}$ fails the Lipschitz condition on the line $y+x=0$ but has unique solutions through any point on this line. Also the vector DE's

$$
d(x, y) / d t=\left(1, y^{\frac{1}{2}}\right) \text { and } d(x, y) / d t=\left(y^{\frac{1}{2}}, 1\right)
$$

both fail the vector Lipschitz condition on the plane $y=0$ in $(t, x, y)$ space and one has unique solutions while the other has indeterminate solutions starting from any point in the above plane.

There are stronger Lipschitz conditions, a selection of which are described by Hille [1], but none of these considers the cases where the solution curve is not tangent to the surface on which the Lipschitz condition fails. The following theorem deals with this property and is used to distinguish between the above simple examples.

THEOREM. Let $\mathrm{F}=\left(1, F_{1}, F_{2}, \ldots, F_{n}\right): V \rightarrow R$ be a continuous vector function defined on an open set $V$ in $R^{n+1}$. Let $a \in V$, let $\pi_{i} \quad(i=1,2, \ldots, r ;(r \leq n))$ be sections of $V$ by surfaces through 
$\mathrm{a}$, and let the neighbourhood of a on one side of each $\pi_{i}$ be $B$ : $\|\mathrm{x}-\mathrm{a}\|<b$. Suppose that in $B$

(i) $F(x)$ is continuous;

(ii) there exist unit normals ${ }_{i}$ to $\pi_{i}$ at a directed into $B$;

(iii) $z_{i}=(\mathrm{x}-\mathrm{a}) \cdot 1_{i}$ is positive;

(iv) $\omega_{i}\left(z_{i}\right)$ are monotonic positive increasing continuously differentiable functions with $\omega_{i}(0)=0$;

(v) $\left|F_{i}(\mathrm{x})-E_{i}(\mathrm{y})\right| \leq d \omega_{i}\left(z_{i}\right) / d z_{i}(\mathrm{x}-\mathrm{y}) \cdot 1_{i}+M\|\mathrm{x}-\mathrm{y}\|$ where $(x-y) \cdot 1_{i} \geq 0$;

(vi) $\left|F_{j}(\mathrm{x})-F_{j}(\mathrm{y})\right| \leq M\|\mathrm{x}-\mathrm{y}\|, j=r+1, \ldots, n$;

(vii) $1_{i} \cdot \mathrm{F} \geq m$.

Then the vector $\mathrm{DE} d \mathrm{x} / d x_{0}=F(x)$ has a unique differentiable solution $x=f\left(x_{0}\right)$ in $B$ with $a=f\left(a_{0}\right)$.

Proof. Since $F$ is continuous, then one solution,

$$
x=a+\int_{a_{0}}^{x_{0}} F(x) d x_{0},
$$

exists.

If $f$ and $g$ are any two solutions of the $D E$ through $a$, we set

$$
h_{0}(t)=\left\|f\left(a_{0}+t\right)-g\left(a_{0}+t\right)\right\| ;
$$

hence on integrating the $\mathrm{DE}$

$$
h_{0}(t) \leq \int_{0}^{t}\left\|F\left(f\left(a_{0}+s\right)\right)-F\left(g\left(a_{0}+s\right)\right)\right\| d s,
$$

so by the triangle inequality 


$$
\begin{aligned}
h_{0}(t) \leq \sum_{i=1}^{r} \int_{0}^{t}\left|F_{i}\left(f\left(a_{0}+s\right)\right)-F_{i}\left(g\left(a_{0}+s\right)\right)\right| d s & \\
& +\sum_{j=r+1}^{n} \int_{0}^{t}\left|F_{j}\left(f\left(a_{0}+s\right)\right)-F_{j}\left(g\left(a_{0}+s\right)\right)\right| d s .
\end{aligned}
$$

Now $(f-g) .1_{i} \leq\|f-g\|$ and $d z_{i}=1_{i} . d x=1_{i} . F d x_{0} \geq m d s$ by conditions (iii) and (vii) where $z_{i}$ is the larger of $(f-a) . l_{i}$ and $(g-a) . l_{i}$ for each $i$. Therefore, using conditions $(v)$ and $(v i)$,

$$
h_{0}(t) \leq \sum_{i=1}^{r} \int_{0}^{t} \frac{1}{m} h_{0}(s) \frac{d w_{i}\left(z_{i}\right)}{d z_{i}} \frac{d z_{i}}{d s} d s+n M \int_{0}^{t} h_{0}(s) d s .
$$

Hence from condition ( $i v)$ and a simple extension of Hille's [1] theorems 1.5.1 and $1.5 .2^{1}$ to the sum of several integrals, this inequality has only one solution $h_{0}(t) \equiv 0$. Hence $f=g$, so the solution is unique in $B$.

COMMENTS. It may be seen in the proof that the factor $(x-y) .1_{i}$ in condition ( $v$ ) could have been replaced by $\|x-y\|$ but the form given is that in which this condition frequently occurs.

If $d \omega_{i} / d z_{i}$ is bounded, we have a simple Lipschitz condition, but when it is unbounded, condition (vii) provides a sufficient condition for uniqueness. Condition (vii) ensures that $F$ is not tangent to the surfaces $\pi_{i}$.

In order to determine any surfaces $\pi_{i}$ and their normals $i_{i}$ on which $d \omega_{i}\left(z_{i}\right) / d z_{i}$ is unbounded we may observe that for some simple differentiable functions $F_{i}(x)$, if $\operatorname{grad} F_{i}$ is unbounded on a surface $\pi_{i}$ then $1_{i}=\lim \operatorname{grad} F_{i} /\left\|\operatorname{grad} F_{i}\right\|$ as $d\left[x, \pi_{i}\right] \rightarrow 0$. Also for simple $F_{i}$, $d \omega_{i}\left(z_{i}\right) / d z_{i}$ may be related to $\left\|\operatorname{grad} F_{i}\right\|$.

\footnotetext{
1 Briefly 1.5.1 $f(t) \leq k \int_{0}^{t} f(s) d s$ and 1.5 .2 $f(t) \leq \int_{0}^{t} K(s) f(s) d s=f(t) \equiv 0$.
} 
In applying the theorem to the second $\mathrm{DE}$ of the introduction, $x=(x, y)$ and $F_{1}=(x+y)^{\frac{1}{2}}$ so $\operatorname{grad} F_{1}=\frac{1}{2}(x+y)^{-\frac{1}{2}}(1,1)$ which is unbounded on $\pi: x+y=0$ so $1=(1,1) 2^{-\frac{1}{2}}$ and $F .1=2^{-\frac{1}{2}}$ on $\pi$ while $\omega(z)=2^{\frac{5}{4}} z^{\frac{1}{2}}$. All conditions apply for $(x+y)>0$ so a solution through $(a,-a)$ is unique.

If the third equation is written as $d(t, x, y) / d t=\left(1,1, y^{\frac{1}{2}}\right)$, then $\operatorname{grad} F_{2}$ is unbounded on $\pi: y=0$ where $1=(0,0,1)$ so $\mathrm{F} .1=y^{\frac{1}{2}}=0$ on $\pi$ so the theorem does not apply. For the fourth $\mathrm{DE}$ $F .1=1$ so the theorem applies.

\section{REFERENCE}

[1] Einar Hille, Lectures on ordinary differential equations

(Addison-Wesley, Reading, Massachusetts; Menlo Park, California; London; Don Mills, Ontario; 1969).

Australian National University, Canberra, ACT. 